\title{
Carbapenem-Resistant Klebsiella pneumoniae influences the outcome of early infections in liver transplant recipients
}

\author{
Francesco Barchiesi ${ }^{1^{*}}$ (D), Roberto Montalti ${ }^{2}$, Pamela Castelli ${ }^{1}$, Daniele Nicolini ${ }^{2}$, Silvia Staffolani ${ }^{1}$, \\ Federico Mocchegiani ${ }^{2}$, Alessandro Fiorentini ${ }^{1}$, Esther Manso ${ }^{3}$ and Marco Vivarelli ${ }^{2}$
}

\begin{abstract}
Background: Infections remain a leading cause of morbidity and mortality among liver transplant (LT) recipients. The aim of our study was to define the factors associated with outcome of early bacterial and fungal infections in a cohort of patients who underwent LT at the University Hospital of Ancona over a nine year period.

Methods: All consecutive patients who underwent LT in our center were considered. An early infection was defined as occurring in the first month post-transplantation.

Results: Among 330 patients who underwent LT from August 2005 to October 2014, 88 (27 \%) had at least one infection documented within 30 days after transplantation. In 54 cases only one site was involved, in 34 cases $\geq 2$ sites. There were 43 (30\%) pneumonia, 40 (27\%) surgical site infections, 31 (22\%) blood stream infections, and 30 (21\%) urinary tract infections. Gram-negative bacteria accounted for $64 \%$ of the culture-positive cases, followed by Gram-positive bacteria (30\%) and fungi (6\%). A high proportion of drug-resistant strains was found within either Gram-negative (79\%) or Gram-positive (81\%) bacteria. There were 27 out 88 patients (31\%) who died within 180 days from the transplant. Factors independently associated with a higher risk of mortality were: renal replacement therapy (HR 11.797 [Cl95 \% 3.082-45.152], $p<0.0001$ ), multisite infections (HR 4.865 [C195 \% 1.417-16.700], $p=0.012$ ) and being infected with carbapenem-resistant Klebsiella pneumoniae (CRKP; HR 5.562 [C195 \% 1.186-26.088], $p=0.030$ ).

Conclusions: Overall, these data indicate that early infections in LT patients are characterized by significant mortality. In particular, an early infection caused by CRKP has an adverse impact on survival in these patients suggesting an urgent need for adopting preventive measures to avoiding this complication.
\end{abstract}

Keywords: Liver transplantation, Early infections, Antibiotic resistance, Carbapenem-resistant Klebsiella pneumonaie, Immunosuppression

\section{Background}

Infections remain a leading cause of morbidity and mortality among liver transplant (LT) recipients [1]. Bacterial infections are the most frequent type of infectious diseases post-transplant, followed by fungal, viral and protozoal infections [2]. A vast majority of bacterial infections occur within the first month after transplantation and most of these are caused by nosocomial organisms [3].

\footnotetext{
*Correspondence: f.barchiesi@univpm.it

${ }^{1}$ Clinica Malattie Infettive, Università Politecnica delle Marche, Azienda Ospedaliero-Universitaria, Ospedali Riuniti Umberto I'-Lancisi-Salesi, Via Conca, 60126 Ancona, Italy

Full list of author information is available at the end of the article
}

Accumulating data in the last several years has documented a shift towards increase in Gram-negative bacterial infections and the emergence of multi-drug-resistant (MDR) bacterial pathogens [4]. In particular, recent reports that have investigated the impact of carbapenemaseresistant- Klebsiella pneumonia (CRKP) in LT-recipients have documented a mortality rate up to $70 \%$ [5]. These data underscore the importance of rigorous infection control practices to curtail the spread of resistant bacteria which are particularly difficult to manage and are associated with poor outcomes in these patients.

The aim of our study was to analyze the factors related to outcome of bacterial and fungal infections in 
the early post-transplant period in a cohort of patients undergoing LT.

\section{Methods Study design}

This was a retrospective, observational study conducted at the Università Politecnica delle Marche, Ancona, Italy from August 2005 to October 2014. The patient population included all patients who underwent orthotopic LT and who survived more than $48 \mathrm{~h}$ after transplantation. The study group consisted of those patients who developed an early bacterial or fungal infection after LT. Demographic, microbiological and clinical characteristics, including preoperative, intraoperative and postoperative recipient variables, were collected and 180-day mortality from the transplant date was calculated. The present research has been performed in accordance with the ethical standards of the 1964 Declaration of Helsinki and its later amendements. The Institutional Review Board of the Azienda Ospedaliero-Universitaria Ospedali Riuniti Umberto $I^{\circ}$-Lancisi-Salesi granted retrospective access to the data without need for individual informed consent.

\section{Definition and microbiology}

An early infection was defined as that occurring in the first month post-transplantation. Infections were identified through active surveillance in the LT ward, and through reviews of outpatient medical records. The criteria used for defining and classifying infections were those proposed by the Centers for Disease Control and Prevention [6]. In particular, the following were considered: pneumonia, surgical site infections (SSIs, including deep intra-abdominal infections), blood stream infections (BSIs, including vascular catheter-related infections) and urinary tract infections (UTIs). Cultures for the diagnosis of bacterial or fungal infection in blood, sputum (or other respiratory secretions), urine or ascitic fluid were obtained on the basis of clinical suspicion as standard of care. Isolation of an organism from nonsterile body sites such as drainage catheters in the absence of clinical signs of infection was considered colonization. Microorganisms were cultured and identified according to standard bacteriological procedures. Susceptibility testing of the strains to antibacterial agents were performed by standard methods and the patterns were reviewed and classified according to the ESCMID (European Society for Clinical Microbiology \& Infectious Diseases) guidelines [7]. In particular, MDR was defined as non-susceptibility to at least one agent in three or more antimicrobial categories; XDR (extensively drug-resistant) was defined as non-susceptibility to at least one agent in all but two or fewer antimicrobial categories; PDR (pandrug-resistant) was defined as non-susceptibility to at all agents in all antimicrobial categories.

\section{Statistical analysis}

Patients were categorized into two subgroups based on outcome (death or survival) at 180-day from the LT. Quantitative data are depicted as median with interquartile (Q1- Q3) ranges and compared by U Mann-Whitney test. Qualitative variables were expressed as absolute and relative frequencies. Categorical variables were compared using the $\mathrm{X}^{2}$ test with Yates' correction or Fisher's exact test when appropriate. The diagnostic accuracy of selected risk factors was evaluated using receiver operating curve (ROC). We analyzed the factors associated with mortality by using a stepwise binary logistic regression model in which variables found to be significant at the univariate level $(P$-value $<0.05)$ were introduced. Statistical analysis was performed using SPSS software, version 20 (Statistical Package for Social Sciences Inc., Chicago, IL).

\section{Results}

Among 330 LT patients considered in the study period, $88(27 \%)$ had at least one infection documented within 30 days after the transplant. Demographic and clinical characteristics of these patients are reported in Table 1. Median age was 53 years, male accounted for $80 \%$ of the population. The majority of the patients (66\%) underwent LT due to a viral infection (HCV $56 \%$ [49/88] and HBV $15 \%$ [13/88]). There were 9 patients HIV-coinfected. The type of anastomosis was termino-terminal in $89 \%$ of the patients. Twenty-three percent of the patients underwent renal-replacement-therapy (RRT) in the peri-operative period.

In 54 out of 88 (61\%) patients only one site was involved while in 34 cases $(39 \%) \geq 2$ sites. A total of 144 infections were documented. There were 43 (49 \%) pneumonia, 40 (45\%) SSIs, 31 (35\%) BSIs and 30 (34 \%) UTIs. Gram-negative bacteria accounted for $64 \%$ of the culture-positive cases, followed by Gram-positive bacteria (30\%) and fungi (6\%). Polymicrobial and mixed (bacterial plus fungal) infections occurred in $27 \%$ and $7 \%$ of the patients, respectively. Infections were due to drug-resistant bacteria in 78 (89\%) of the patients.

Pathogens isolated in the study cohort are shown in Table 2. Among Gram-negative bacteria, the most frequently isolated pathogen was Klebsiella pneumoniae (29 \%) followed by Pseudomonas aeruginosa (22\%) and Escherichia coli (21 \%) while among Gram-positive bacteria the most frequent organism was Enterococcus faecium (57 \%) followed by Staphylococcus aureus (19\%) and Staphylococcus epidermidis (9\%).

Of 112 Gram-negative bacteria, 88 isolates (79\%) were antibiotic resistant organisms and included 51 MDR and 
Table 1 Demographic and clinical characteristics of the study cohort

\begin{tabular}{|c|c|c|c|c|}
\hline \multirow[t]{2}{*}{ Characteristics } & \multirow{2}{*}{$\begin{array}{l}\text { All patients } \\
n=88,(\%)\end{array}$} & \multicolumn{2}{|c|}{ 180-day outcome } & \multirow[t]{2}{*}{$p$ value } \\
\hline & & $\begin{array}{l}\text { Survival } \\
n=61(\%)\end{array}$ & $\begin{array}{l}\text { Death } \\
n=27(\%)\end{array}$ & \\
\hline Age (years, ranges) & $53(34-67)$ & $52(34-66)$ & $54(37-67)$ & 0.803 \\
\hline Gender Male & $70(80)$ & $51(84)$ & $19(70)$ & 0.257 \\
\hline MELD score $\geq 25^{\mathrm{a}}$ & $23(26)$ & $14(23)$ & $9(33)$ & 0.448 \\
\hline Child-Pugh stage $C$ & $42(48)$ & $27(44)$ & $15(56)$ & 0.436 \\
\hline Pre-LT hospitalization ${ }^{\mathrm{b}}$ & $18(20)$ & $10(16)$ & $8(30)$ & 0.257 \\
\hline Previous Abdominal Surgery & $7(8)$ & $5(8)$ & $2(7)$ & 0.900 \\
\hline Indication for $L T$ - Viral ${ }^{c}$ & $58(66)$ & $40(66)$ & $18(67)$ & 1.000 \\
\hline HCV positivity & $49(56)$ & $35(57)$ & $14(52)$ & 0.804 \\
\hline HBs-Ag positivity & $13(15)$ & $8(13)$ & $5(18)$ & 0.739 \\
\hline HIV positivity & $9(10)$ & $5(8)$ & $4(15)$ & 0.448 \\
\hline Presence of $\mathrm{HCC}^{d}$ & $30(34)$ & $22(36)$ & $8(30)$ & 0.731 \\
\hline \multicolumn{5}{|l|}{ Type of anastomosis } \\
\hline Roux-en-y & $10(11)$ & $6(10)$ & $4(15)$ & 0.488 \\
\hline Termino-terminal & $78(89)$ & $55(90)$ & $23(85)$ & \\
\hline RBC units $\geq 5^{e}$ & $72(82)$ & $48(79)$ & $24(89)$ & 0.398 \\
\hline Plasma units $\geq 10$ & $39(44)$ & $26(43)$ & $13(48)$ & 0.804 \\
\hline $\operatorname{RRT}^{f}$ & $20(23)$ & $5(8)$ & $15(56)$ & $<0.0001$ \\
\hline Rejection & $41(47)$ & $30(49)$ & $11(41)$ & 0.617 \\
\hline Diabetes & $14(16)$ & $12(20)$ & $2(7)$ & 0.216 \\
\hline Multisite infections ${ }^{g}$ & $34(39)$ & $14(23)$ & $20(74)$ & $<0.0001$ \\
\hline Pneumonia & $43(49)$ & $22(36)$ & $21(78)$ & 0.001 \\
\hline SSIs $\mathrm{h}^{\mathrm{h}}$ & $40(45)$ & $25(41)$ & $15(56)$ & 0.301 \\
\hline BSIs ${ }^{\mathrm{i}}$ & $31(35)$ & $14(23)$ & $17(63)$ & 0.001 \\
\hline UTIS ${ }^{j}$ & $30(34)$ & $20(33)$ & $10(37)$ & 0.885 \\
\hline Gram-positive bacteria & $45(51)$ & $29(48)$ & $16(59)$ & 0.434 \\
\hline Gram-negative bacteria & $64(73)$ & $40(66)$ & $24(89)$ & 0.045 \\
\hline Polymicrobial infections ${ }^{k}$ & $24(27)$ & $11(18)$ & $13(48)$ & 0.008 \\
\hline Fungi & $9(10)$ & $5(8)$ & $4(15)$ & 0.448 \\
\hline Mixed infections' & $6(7)$ & $2(3)$ & $4(15)$ & 0.069 \\
\hline Overall resistant infections ${ }^{m}$ & $78(89)$ & $52(85)$ & $26(96)$ & 0.253 \\
\hline CRKP infections $^{n}$ & $13(15)$ & $4(7)$ & $9(33)$ & 0.002 \\
\hline CMV infection & $29(33)$ & $14(23)$ & $15(56)$ & 0.006 \\
\hline
\end{tabular}

${ }^{a}$ MELD: Model for End-Stage Liver Disease

${ }^{b}$ Pre-LT hospitalization: included any hospitalization within one month before LT

Indication for LT included: viral $[n=58]$, alcoholic $[n=11]$, cryptogenetic $[n=7]$, cholestatic $[n=6]$, and other [ $n=6]$ causes

${ }^{\mathrm{d}} \mathrm{HCC}:$ Hepato Cellular Carcinoma

${ }^{\mathrm{e}} \mathrm{RBC}$ : red blood cell units

fRRT: renal replacement therapy included: dialysis, continuous veno-venous haemo(dia)filtration and plasmapheresis

${ }^{9}$ Multisite infections: $\geq 2$ sites (i.e.: blood and urine; blood and surgical sites etc.) were contemporarily involved

hSSIs: surgical sites infections

'BSIs: blood stream infections

'UTIs: urinary tract infections

${ }^{k}$ Polymicrobial infections: infections caused by both Gram-negative and Gram-positive bacteria

'Mixed infections: infections caused by both bacterial and fungal pathogens

moverall resistant infections: infections caused by a bacterial pathogen showing any resistant pattern (see for details Table 3)

${ }^{n}$ CRKP infections: infections caused by carbapenem-resistant Klebsiella pneumoniae strains 
Table 2 Pathogens isolated in the study cohort

\begin{tabular}{|c|c|c|c|c|c|c|}
\hline \multirow[t]{2}{*}{ Microorganisms $^{a}$} & \multirow[t]{2}{*}{$\mathrm{n}^{\circ}(\%)$} & \multicolumn{4}{|c|}{ Types of infection ${ }^{b}$} & \multirow[t]{2}{*}{ Susceptibility patterns (\%) } \\
\hline & & Pneumonia & SSIs & BSIs & UTIS & \\
\hline \multirow[t]{3}{*}{ K. pneumoniae } & $33(29)$ & 11 & 9 & 7 & 6 & $\begin{array}{l}22 \text { CRKP (67) } \\
2 \text { ESBL (6) } \\
4 \text { MDR (12) } \\
1 \text { XDR (3) }\end{array}$ \\
\hline & & & & & & $1 \mathrm{R}(3)$ \\
\hline & & & & & & $3 S(9)$ \\
\hline P. aeruginosa & $25(22)$ & 14 & 6 & 2 & 3 & $\begin{array}{l}5 \mathrm{MDR}(20) \\
14 \mathrm{R}(56) \\
6 \mathrm{~S}(24)\end{array}$ \\
\hline E. coli & $23(21)$ & 8 & 2 & 4 & 9 & $\begin{array}{l}4 \text { ESBL (17) } \\
14 \text { MDR (61) } \\
5 \text { S (22) }\end{array}$ \\
\hline S. maltophilia & $7(6)$ & 5 & 1 & 1 & - & $\begin{array}{l}1 R(14) \\
6 S(86)\end{array}$ \\
\hline A. baumannii & $4(4)$ & 3 & - & 1 & - & $\begin{array}{l}1 \mathrm{MDR}(25) \\
1 \mathrm{XDR}(25) \\
2 \mathrm{R}(50)\end{array}$ \\
\hline Other gram-neg. & $20(18)$ & 4 & 3 & 6 & 7 & $\begin{array}{l}5 \mathrm{MDR}(25) \\
1 \mathrm{ESBL}(5) \\
10 \mathrm{R}(50) \\
4 \mathrm{~S}(20)\end{array}$ \\
\hline \multirow[t]{2}{*}{ E. faecium } & $30(57)$ & 1 & 20 & 7 & 2 & $28 \mathrm{MDR}(94)^{d}$ \\
\hline & & & & & & $\begin{array}{l}1 \mathrm{VRE}(3) \\
1 \mathrm{R}(3)\end{array}$ \\
\hline S. aureus & $10(19)$ & 4 & 5 & 1 & - & $\begin{array}{l}4 \text { MRSA (40) } \\
6 \mathrm{~S}(60)\end{array}$ \\
\hline S. epidermidis & $5(9)$ & - & 1 & 3 & 1 & 5 MRSE (100) \\
\hline Other gram-pos. & $8(15)$ & 3 & 3 & - & 2 & $\begin{array}{l}6 \mathrm{MDR}(75) \\
2 \mathrm{~S}(25)\end{array}$ \\
\hline C. albicans & $4(40)$ & - & 1 & - & 3 & ND \\
\hline C. glabrata & $3(30)$ & - & 2 & 1 & - & ND \\
\hline C. tropicalis & $2(20)$ & - & - & - & 2 & ND \\
\hline C. dubliniensis & $1(10)$ & - & 1 & - & - & ND \\
\hline
\end{tabular}

${ }^{a}$ Others included: Gram negative bacteria, Enterobacter cloacae $\left(n^{\circ} 5\right)$, Enterobacter aerogenes $\left(n^{\circ} 1\right)$, Serratia marcescens $\left(n^{\circ} 4\right)$, Klebsiella oxytoca $\left(n^{\circ} 2\right)$, Acinetobacter iwoffii $\left(n^{\circ} 2\right)$, Acinetobacter junii $\left(n^{\circ} 1\right)$, Haemophilus influenzae $\left(n^{\circ} 1\right)$, Citrobacter braaki $\left(n^{\circ} 1\right)$, Prevotella spp. $\left(n^{\circ} 1\right)$, Bacteroides uniformis $\left(n^{\circ} 1\right)$, Morganella morganii $\left(n^{\circ} 1\right)$; Gram positive bacteria: Enterococcus faecalis $\left(n^{\circ} 2\right)$, Streptococcus pneumoniae $\left(n^{\circ} 2\right)$, Staphylococcus haemolyticus $\left(n^{\circ} 2\right)$, Staphylococcus pseudointermedius $\left(n^{\circ} 1\right)$, Staphylococcus spp. $\left(n^{\circ} 1\right)$

${ }^{\mathrm{b}} \mathrm{SSIS}$, surgical site infections; BSIs, blood stream infections; UTIs, urinary tract infections

'CRKP, carbapenem-resistant Klebsiella pneumoniae; ESBL, extended-spectrum beta-lactamase; XDR, extensive drug resistant; MDR, multi drug resistant; MRSA, methicillin-resistant Staphylococcus aureus; MRSE, methicillin-resistant Staphylococcus epidermidis; VRE, vancomycin-resistant Enterococcus; R, resistance patterns not included in previous definition; S, fully susceptible; -, none; ND, not done; ${ }^{d}$, there was one VRE strain within the 28 MDR

two XDR. Within the former group of isolates there were 22 carbapenem-resistant K. pneumoniae (CRKP) and 7 extended-spectrum beta-lactamase-producing (ESBL) bacteria. Among 53 Gram-positive bacteria, there were 43 MDR isolates (81\%), including 5 methicillin-resistant $S$. epidermidis (MRSE) and 4 methicillin-resistant $S$. aureus (MRSA). Additionally, there were 2 vancomycin-resistant Enterococcus (VRE) isolates one of which was also MDR. Candida albicans was the most commonly isolated yeasts (40\%) followed by Candida glabrata (30\%).

There were 27 out 88 patients (31\%) who died within 180 days from the transplant. The main cause of death was an infectious complication in $67 \%$ of the cases, while vascular, neurological and other types of complications occurred, as main causes of death, in $19 \%, 7 \%$ and $7 \%$ of the cases, respectively. A significantly higher proportion of patients who died within this time interval underwent RRT (Table 1). Additionally, CMV infection, pneumonia, BSIs and multisite infections were all associated with a significantly higher proportion of mortality. Similarly, infections due to Gram-negative bacteria, polymicrobial infections and infections caused by CRKP were all associated with a significantly higher proportion of mortality (Table 1). 
In multivariate analysis, factors independently associated with a higher risk of mortality were: RRT (HR 11.797 [CI95 \% 3.082-45.152], $p<0.0001)$, multisite infections (HR 4.865 [CI95 \% 1.417-16.700], $p=0.012$ ) and being infected with CRKP (HR 5.562 [CI95 \% 1.186-26.088], $p=0.030$ ) (Table 3).

\section{Discussion}

Solid organ transplant recipients are prone to healthcareassociated infections [3]. Liver transplant recipients are especially susceptible to bacterial infections as a result of technical complexity of the surgical procedure and complications related to abdominal surgery and manipulation of the hepatobiliary system [1-3]. In this study we evaluated the factors related to outcomes of early bacterial and fungal infections in LT patients. Among 330 patients transplanted over a nine year period, 88 (27 \%) had at least one infection documented within 30 days after the transplant and 27 of them (31 \%) died within 180 days from the transplant. Although we investigated the contribution of as many as 30 demographic, clinical and microbiological characteristics, only three of these factors were independently associated with a higher risk of mortality. These included RRT, multisite infections, and infection due to CRKP.

Although only a minority of patients in our series (22/88) underwent RRT during the intra- and peri-operative period, this variable was significantly associated with a negative outcome. Of note, the majority of our patients undergoing this procedure were suffering from acute kidney failure which is a known risk factor for higher mortality in LT-recipients [8].

The type of infection per se did not influence significantly the outcome. Pneumonia and BSIs, which are generally characterized by negative outcomes in immunocompromised patients, showed to be significantly associated with high mortality rate at univariate analysis while their effect was lost in the multivariate model $[9,10]$. Indeed multisite infections remained independently associated with higher risk of mortality. This

Table 3 Multivariate analysis of risk factors for 180-day mortality of the study cohort

\begin{tabular}{lllll}
\hline Risk factors & Hazard ratio & Cl 95 \% & \multirow{2}{*}{$p$ value } \\
\cline { 3 - 4 } & & Lower limit & Upper limit & \\
\hline RRT $^{\mathrm{a}}$ & 11.797 & 3.082 & 45.152 & $<0.0001$ \\
Multisite infections $^{\mathrm{b}}$ & 4.865 & 1.417 & 16.700 & 0.012 \\
CRKP infections $^{c}$ & 5.562 & 1.186 & 26.088 & 0.030 \\
\hline
\end{tabular}

${ }^{a}$ RRT: renal replacement therapy included: dialysis, continuous veno-venous haemo(dia)filtration and plasmapheresis

${ }^{b}$ Multisite infections: $\geq 2$ sites were contemporarily involved ${ }^{\mathrm{C} C R K P}$ infections: infections caused by carbapenem-resistant Klebsiella pneumoniae strains can be due to the fact that involvement of two or more sites is typical of sicker patients.

As a consequence of numerous hospitalizations, invasive procedures, and frequent use of antibiotics LT, patients accumulate risk factors for infections with drugresistant organisms [1-3]. In our series, almost $90 \%$ of the patients suffered from early infections caused by drug-resistant bacteria. Importantly, we found that infection due to CRKP was one of the strongest predictor of post-LT mortality. This finding agrees with that recently reported by two studies showing that 1-year survival dramatically dropped from $86 \%$ to $29 \%$ and from $93 \%$ to $55 \%$ when LT patients were infected with CRKP $[11,12]$. Although several therapeutic approaches have been experimented for CRKP infections including combination regimens which are usually associated with a lower risk of mortality, the management of these infections is still extremely difficult mainly in the immunocompromised host [11-15]. Overall, these data suggest that in an endemic area improved strategies for screening and prevention of CRKP infections are urgently needed. One prospective study conducted on 237 LT patients found that RRT, mechanical ventilation $>48 \mathrm{~h}, \mathrm{HCV}$ recurrence and colonization with CRKP at any time (i.e.: before and after LT) were all independent risk factors for CRKP infections [16]. Interestingly, based on these four variables the authors developed a risk score able to discriminate patients at low $v s$ higher risk for CRKP infections [16]. Besides these essential preventive strategies, it is crucial to introduce into the market new effective antibiotics especially for those patients, such as transplant-candidates and -recipients, in which the presence of a difficult-to-treat bacterial infection may either retard a life-saving procedure or worsen an already fragile postoperative course.

The present study has several limitations. First, this was a retrospective analysis. Although we tried to collect as many clinical data as possible, we may have still missed useful information for the management of LTpatients. Second, we limited our observation to those infections occurring within one month post-OLT. This time interval was selected given that patients are at highest risk for infections during this period. Further studies addressing the prevalence of infections over a longer period of time post-OLT are ongoing in our center. Third, since our data come from a single-center experience with LT recipients belonging to an area endemic for CRKP, our findings may not be relevant to other patients populations.

\section{Conclusion}

In conclusion, these data indicate that early infections in LT patients are characterized by significant mortality. In particular, an early infection caused by CRKP has an adverse impact on survival in these patients. 


\section{Abbreviations}

BSI: Blood stream infections; Cl: Confidence interval; CMV: Cytomegalovirus; CRKP: Carbapenem-resistant Klebsiella pneumoniae; ESBL: Extended-spectrum beta-lactamase; ESCMID: European society for clinical microbiology \& infectious diseases; HCC: Hepato cellular carcinoma; HR: Hazard ratio; LT: Liver transplantation; MDR: Multi drug-resistant; MELD: Model for end-stage liver disease; MRSA: Methicillin-resistant S. aureus; MRSE: Methicillin-resistant S. epidermidis; OLT: Orthotopic liver transplantatation; PDR: Pandrug resistant; Q1-Q3: Interquartile ranges; R: Resistant; S: Susceptible; RBC: Red blood cell units; ROC: Receiver operating curve; RRT: Renal replacement therapy; SSIs: Surgical site infections; UTIs: Urinary tract infections; VRE: Vancomycinresistant Enterococcus; XDR: Extensively drug-resistant

\section{Acknowledgements}

Not applicable.

\section{Funding}

Not applicable.

\section{Availability of data and materials}

The data cannot be shared as local Institutional Review Board has no policy to share the data without prior permission.

\section{Authors' contributions}

Conceived and designed the experiments: FB RM PC SS MV. Performed the experiments: RM DN. FM PC SS AF. Analyzed the data: FB RM DN. Contributed reagents/materials/analysis tools: EM. Wrote the paper: FB MV. All authors drafted the article, revised it critically for important intellectual content, and approved the final article.

\section{Competing interests}

The authors report no competing interests. The authors alone are responsible for the content and the writing of the paper.

\section{Consent for publication}

\section{Not applicable.}

\section{Ethics approval and consent to participate}

The present research has been performed in accordance with the ethical standards of the 1964 Declaration of Helsinki and its later amendements. The Institutional Review Board of the Azienda Ospedaliero-Universitaria Ospeadali Riuniti Umberto ${ }^{\circ}$-Lancisi-Salesi granted retrospective access to the data without need for individual informed consent.

\section{Author details}

${ }^{1}$ Clinica Malattie Infettive, Università Politecnica delle Marche, Azienda

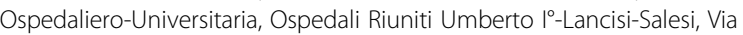
Conca, 60126 Ancona, Italy. ${ }^{2}$ Chirurgia Epatobiliare e dei Trapianti, Università Politecnica delle Marche, Azienda Ospedaliero-Universitaria, Ospedali Riuniti Umbero I'-Lancisi-Salesi, Ancona, Italy. ${ }^{3}$ Laboratorio di Microbiologia, Azienda Ospedaliero-Universitaria, Ospedali Riuniti Umberto Iº-Lancisi-Salesi, Ancona, Italy.

Received: 6 July 2016 Accepted: 27 September 2016

Published online: 04 October 2016

\section{References}

1. Kim SI. Bacterial infection after liver transplantation. World J Gastroenterol. 2014;20:6211-20.

2. Blair JE, Kusne S. Bacterial, mycobacterial, and protozoal infections after liver transplantation-part I. Liver Ttransplant. 2005;11:1452-9.

3. Fishman JA. Infections in immunocompromised hosts and organ transplant recipients: essentials. Liver Transplant. 2011;17 Suppl 3:S34-7.

4. Singh N, Wagener MM, Obman A, et al. Bacteremias in liver transplant recipients: shift toward gram-negative bacteria as predominant pathogens. Liver Transplant. 2004;10:844-9. doi:10.1002/lt.20214.

5. Shi SH, Kong HS, Xu J, et al. Multidrug resistant gram-negative bacilli as predominant bacteremic pathogens in liver transplant recipients. Transplant Infect Dis. 2009;11:405-12.

6. Centers for Disease Control and Prevention. CDC/NHSN surveillance definitions for specific types of infections. 2014; Centers for Disease Control and Prevention, Atlanta, GA. http://www.cdc.gov/nhsn/PDFs/pscManual/ 17pscNosInfDef current.pdf.

7. Magiorakos AP, Srinivasan A, Carey RB, et al. Multidrug-resistant, extensively drug-resistant and pandrug-resistant bacteria: an international expert proposal for interim standard definitions for acquired resistance. Clin Microbiol Infect. 2012;18:268-81.

8. Kawecki D, Pacholczyk M, Lagiewska B, et al. Bacterial and fungal infections in the early post-transplantation period after liver transplantation: etiologic agents and their susceptibility. Transplant Proc. 2014;46:2777-81.

9. Bert F, Larroque B, Paugam-Burtz C, et al. Microbial epidemiology and outcome of bloodstream infections in liver transplant recipients: an analysis of 259 episodes. Liver Transplantat. 2010;16:393-401.

10. Li C, Wen TF, Mi K, et al. Analysis of infections in the first 3-month after living donor liver transplantation. World J Gastroenterol. 2012;18:1975-80.

11. Pereira MR, Scully BF, Pouch SM, et al. Risk factors and outcomes of carbapenem-resistant Klebsiella pneumoniae infections in liver transplant recipients. Liver Transpl. 2015;21:1511-9.

12. Kalpoe JS, Sonnenberg E, Factor SH, et al. Mortality associated with carbapenem-resistant Klebsiella pneumoniae infections in liver transplant recipients. Liver Transpl. 2012;18:468-74.

13. Lübbert C, Becker-Rux D, Rodloff AC, et al. Colonization of liver transplant recipients with $\mathrm{KPC}$-producing Klebsiella pneumoniae is associated with high infection rates and excess mortality: a case-control analysis. Infection. 2014; 42:309-16.

14. Zhong L, Men TY, Li H, et al. Multidrug-resistant gram-negative bacterial infections after liver transplantation - spectrum and risk factors. J Infect. 2012;64:299-310.

15. Clancy CJ, Chen L, Shields RK, et al. Epidemiology and molecular characterization of bacteremia due to carbapenem-resistant Klebsiella pneumoniae in transplant recipients. Am J Transplant. 2013;13:2619-33.

16. Giannella M, Bartoletti M, Morelli MC, et al. Risk factors for infection with carbapenem-resistant Klebsiella pneumoniae after liver transplantation: the importance of pre- and posttransplant colonization. Am J Transplant. 2015; 15:1708-15

\section{Submit your next manuscript to BioMed Central and we will help you at every step:}

- We accept pre-submission inquiries

- Our selector tool helps you to find the most relevant journal

- We provide round the clock customer support

- Convenient online submission

- Thorough peer review

- Inclusion in PubMed and all major indexing services

- Maximum visibility for your research

Submit your manuscript at www.biomedcentral.com/submit
Biomed Central 